\title{
Cardiovascular Toxicity of Novel HER2-Targeted Therapies in the Treatment of Breast Cancer
}

\author{
Susan F. Dent ${ }^{1} \cdot$ Amber Morse $^{1} \cdot$ Sarah Burnette ${ }^{1} \cdot$ Avirup Guha $^{2,3} \cdot$ Heather Moore $^{1}$ \\ Accepted: 1 July 2021 / Published online: 27 August 2021 \\ (c) The Author(s), under exclusive licence to Springer Science+Business Media, LLC, part of Springer Nature 2021
}

\begin{abstract}
Purpose of Review HER2-targeted therapies have led to improved clinical outcomes in early and advanced breast cancer (BC). We review the long-term cardiotoxicity of HER2-targeted therapy in early and advanced BC, our current knowledge of cardiotoxicity of novel HER2-targeted therapies, and propose a cardiac monitoring (CM) strategy for this population.

Recent Findings Long-term data from studies with HER2-targeted therapy in the adjuvant setting have failed to demonstrate an increase in cardiotoxicity over time, and rates of cardiotoxicity seen with novel HER2 agents remain low. Despite over a decade of experience with HER2-targeted therapy, $\mathrm{CM}$ in clinical practice is inconsistent in patients with early $\mathrm{BC}$ and almost non-existent in advanced BC.

Summary Long-term follow-up of clinical trials with HER2-targeted agents in early and advanced BC has failed to demonstrate increased rates of cardiotoxicity over time, attesting to the long-term safety of this class of drugs for the majority of patients, although the long-term cardiac safety of newer HER2 agents in the non-clinical trial setting is largely unknown. We propose CM incorporating clinical history, cardiac imaging, and biomarkers.
\end{abstract}

Keywords Breast cancer · Novel HER2 agents · Cardiotoxicity $\cdot$ Cardiac monitoring

\section{Introduction}

HER2-targeted therapies have led to improved clinical outcomes in patients with early and advanced breast cancer but are associated with an increased risk of cardiotoxicity. In the pivotal early-stage breast cancer trials with trastuzumab-based therapy, cardiac events were reported in $0-3.9 \%$ of patients depending on treatment regimen [1-3]. However, in population-based studies, higher rates of cardiac events have been reported in older patients at 5 years $(3.1 \%<65$ years old vs. $8.9 \% \geq 65)$ [4], and cohort studies have reported asymptomatic drops in left ventricular ejection

This article is part of the Topical collection on Cardio-oncology

Susan F. Dent

susan.dent@duke.edu

1 Duke Cancer Institute, Duke University, DUMC 3446, Durham, NC 27710, USA

2 Harrington Heart and Vascular Institute, Case Western Reserve University, Cleveland, OH, USA

3 Cardio-Oncology Program, Division of Cardiology, The Ohio State University Medical Center, Columbus, OH, USA fraction (LVEF), the clinical significance of which is largely unknown [5]. A retrospective Danish cohort study of more than 8800 women noted a twofold increase in the risk of heart failure at 9 years for those patients treated with anthracyclines and trastuzumab vs. chemotherapy alone, highlighting the importance of understanding the long-term cardiac safety of these drugs in the non-clinical trial setting [6]. A number of novel HER2-targeted agents (e.g., pertuzumab) have been introduced into clinical practice since the introduction of trastuzumab-based therapy over a decade ago. Dual HER2-targeted therapy (pertuzumab/trastuzumab) is now standard of care for neoadjuvant (prior to breast surgery) and adjuvant (after breast surgery) treatment, particularly in the setting of lymph node involvement. There are now multiple lines of HER2-targeted therapy (e.g., T-DM1) for patients with advanced disease, which has resulted in substantial improvements in progression-free survival and overall survival [7], but what are the long-term cardiovascular consequences of HER2-targeted therapy, particularly for patients with early-stage disease? Furthermore, are novel HER2-targeted agents more cardiotoxic? This paper reviews the long-term cardiotoxicity of HER2-targeted therapy reported in early and advanced breast cancer trials, 
our current state of knowledge of cardiotoxicity of novel HER2-targeted therapies, and briefly describes potential cardioprotective strategies. In the era of modifications of health care delivery imposed by the COVID-19 pandemic, we propose a modified cardiovascular monitoring strategy for this patient population.

\section{Long-Term Cardiotoxicity with Trastuzumab-Based Therapy}

Trastuzumab-based therapy is the cornerstone of cancer therapy for patients with HER2 overexpressing breast cancer. Mechanistically, LVEF decline is seen with trastuzumab, given the ErbB2/ErbB4 receptor expression in cardiomyocytes. Growth factor neuregulin-1 (NRG-1) has a protective role against myocardial stress; however, binding of HER2 therapy to the HER2 receptor may disrupt this pathway resulting in cardiotoxicity. Unlike anthracycline chemotherapy, the probability of cardiotoxicity is not associated with cumulative doses of trastuzumab and is generally reversible [7]. Combination therapy of anthracyclines with trastuzumab has shown higher rates of cardiotoxicity, perhaps given the disruption of the NRG-1 pathway and protective effects in the myocardium that has been damaged by anthracycline use. In the pivotal trial, conducted by Slamon and colleagues, of trastuzumab given concurrently with anthracyclines, there was a significant clinical benefit in patients with advanced breast cancer, but unacceptably high rates of heart failure (28\%) [3]. In a retrospective cohort study by Bowles et al., the 5-year cumulative incidence of heart failure and/or cardiomyopathy in women with invasive breast cancer was $12.1 \%$ for trastuzumab alone and $20.1 \%$ in those receiving anthracyclines plus trastuzumab [8]. Subsequent trials modified the delivery of trastuzumab (trastuzumab following anthracyclines), resulting in maintenance of clinical efficacy but with lower rates of cardiotoxicity.

In the early-stage breast cancer setting, long-term follow-up of HER2-targeted trials has failed to demonstrate increased rates of cardiotoxicity over time (Table 1) [1, 2]. In the HERA trial, at a median follow-up of 11 years, primary cardiac endpoints were rare, and there was no statistical difference between treatment groups $(0.1 \%(n=2)$ in the observation group vs. $1 \%(n=18)$ in both the 1 and 2 years $(n=17)$ trastuzumab groups). However, secondary cardiac endpoints occurred more frequently with two years of trastuzumab exposure (7.3\%) compared to 1 year of trastuzumab (4.4\%) and observation (0.9\%) with no improvement in clinical outcomes. In both trastuzumab groups, fewer cardiac endpoints occurred after completing trastuzumab treatment than in the scheduled treatment period [1]. In the BCIRG 006, at a median follow-up of 10.3 years, there was a fivefold increase in the incidence of heart failure (HF) in the anthracycline-containing arm-doxorubicin/cyclophosphamide followed by docetaxel and trastuzumab (AC-TH) arm (2\%) vs. the non-anthracycline arm-docetaxel, carboplatin, trastuzumab (TCH) arm (0.4\%); however, this did change with time. The risk of sustained LVEF decline was more common with AC-TH $(n=206)$ vs. the TCH arm $(n=97)[3,9]$.

APHINITY, a large international phase III randomized trial, demonstrated a small but statistically significant benefit of dual HER2 blockade with trastuzumab and pertuzumab with a chemotherapy backbone vs. trastuzumab based therapy alone, in women with early-stage HER2 positive breast cancer; however, the cardiac event rate was low, with $0.7 \%$ reported in the combination arm vs. $0.3 \%$ in the trastuzumab alone arm [10]. The 6-year analysis presented at the 2019 San Antonio Breast Cancer Symposium continued to demonstrate superior clinical efficacy with dual HER2 blockade, particularly in women with node-positive breast cancer, with only one additional cardiac event reported in this updated analysis [11].

With improved clinical outcomes and overall survival in patients with advanced breast cancer, the long-term cardiotoxicity of HER2-targeted therapy has become increasingly relevant. CLEOPATRA was an international, phase III, double-blind, placebo-control trial which randomized over 800 women with HER2 positive advanced breast cancer to docetaxel, trastuzumab, and pertuzumab or docetaxel, trastuzumab, and placebo. In an end-of-study analysis (99month median follow-up), there was one new case of heart failure (onset at 77 months), which resolved in 34 days. One patient crossed over to the dual HER2 blockade arm and experienced left ventricular systolic dysfunction at 46 months. This end of study analysis failed to demonstrate any long-term cardiac safety concerns with the addition of pertuzumab in this patient population [16].

Similarly in the EMILIA trial, a randomized phase III trial of T-DM1 vs. lapatinib/capecitabine in advanced breast cancer demonstrated, at a median of 47.8-month follow-up in the T-DM1 arm and 41.9 months in the lapatinib/capecitabine arm, a very low incidence of grade 3 or worse cardiac dysfunction in both the lapatinib/capecitabine group ( 3 patients, < 1\%) and the T-DM1 group (one patient, <1\%) [17].

Long-term follow-up of clinical trials with HER2-targeted agents in both early and advanced breast cancer has failed to demonstrate increased rates of cardiotoxicity over time, attesting to the long-term safety of this class of drugs for the majority of patients. In clinical practice, the most common cardiac events reported are asymptomatic drops in LVEF (8-18\%), which vary in incidence depending on treatment and patient-related factors (e.g., age, co-morbidities) $[5,24]$. The majority of patients who do experience drops in LVEF will recover with appropriate modification of cancer therapy. Those patients who do experience symptoms or signs of heart failure tend to do so early in the course 


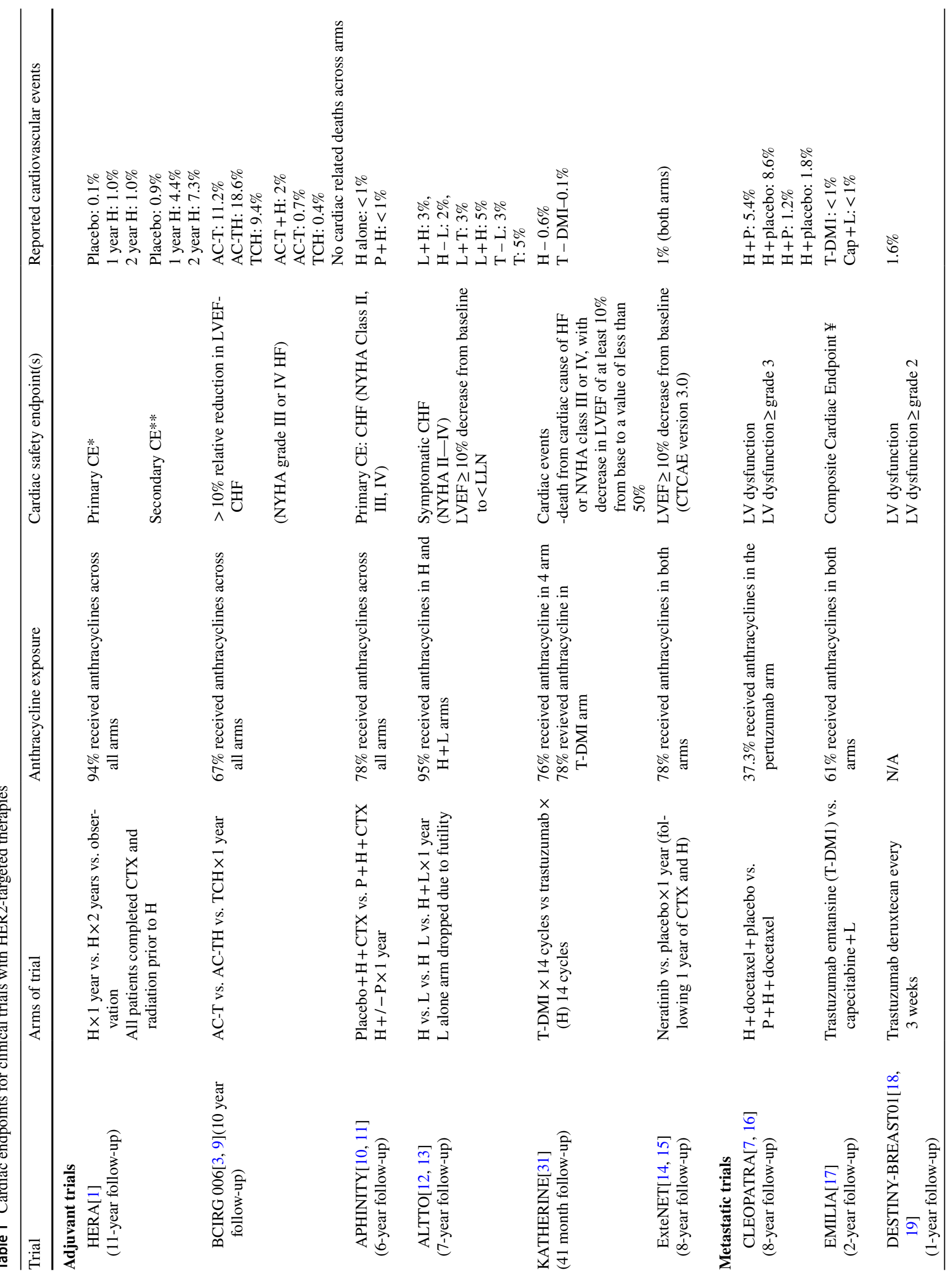




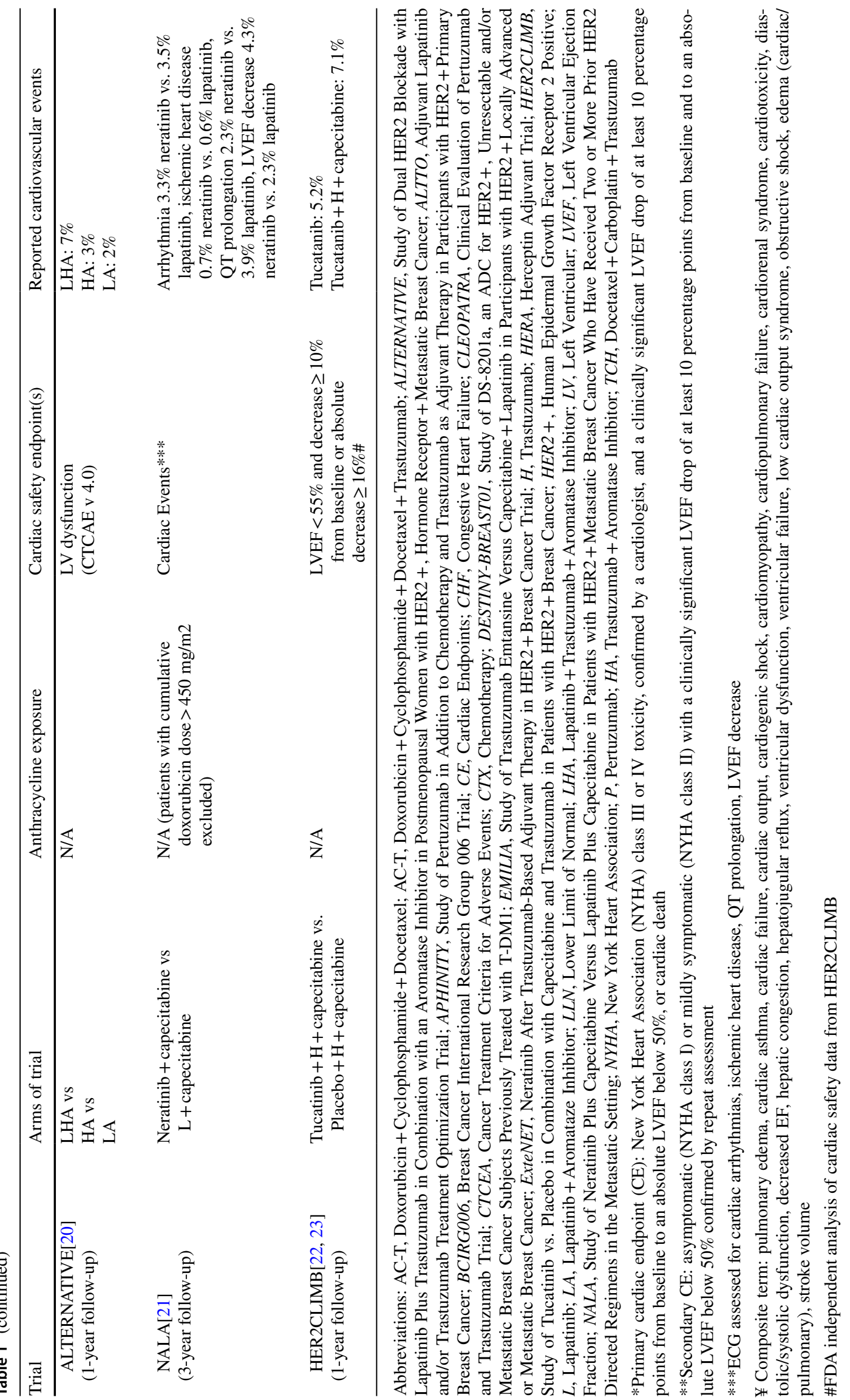


of HER2-targeted therapy. In a real-world study of cardiac events in over 3700 patients with HER2-positive early-stage breast cancer treated with trastuzumab, the incidence of heart failure was $2.8 \%$ with a median onset of 5.7 months and median time to resolution of symptoms of 9.9 months [5]. Identification of this higher-risk population should be the focus of further research, particularly with regards to primary prevention strategies.

\section{Cardiotoxicity of Novel HER2-Targeted Therapies}

The FDA has recently approved several new therapies for advanced HER2-positive breast cancer (Table 2), but little is known about potential long-term cardiovascular toxicities. Here we review the mechanism of action of recently approved HER2-targeted therapies (Fig. 1) and the cardiotoxicity reported in the respective clinical trials.

\section{Antibody-Drug Conjugates (ADCs)}

\section{Fam-Trastuzumab Deruxtecan (DS-8201)}

Fam-trastuzumab deruxtecan (Enhertu) or DS-8201 is an antibody-drug conjugate consisting of trastuzumab and Dxd, an exatecan derivative and topoisomerase inhibitor, with a high chemotherapy payload and a drug to antibody ratio of approximately eight to one respectively. Fam-trastuzumab deruxtecan was approved for HER2-positive, advanced breast cancer in December 2019 based on the DestinyBreast01 phase II clinical trial in which the objective response rate was an impressive $61 \%$ in heavily pretreated patients $[18,30]$.

Given the trastuzumab component, fam-trastuzumab deruxtecan does carry a warning for left ventricular dysfunction, with a recommendation for LVEF assessments at baseline and at regular intervals during treatment. Additional recommendations regarding continuation, discontinuation, or holding of therapy are provided based on the degree or severity of LVEF change [30].

Despite containing trastuzumab, thus far the reported rates of cardiotoxicity associated with fam-trastuzumab deruxtecan have been low. In Destiny-Breast01, a decrease in LVEF occurred in three patients, two grade 2 and one grade 3 , and all were asymptomatic; patients recovered or were recovering after therapy interruption. There were no events of cardiac failure associated with the LVEF decrease [18]. There were no cases of decreased ejection fraction in DESTINY-Breast04 that included HER2 low or equivocal patients [19]. The low cardiotoxicity rates seen with fam-trastuzumab deruxtecan are similar when compared to another antibody-drug conjugate containing trastuzumab, ado-trastuzumab emtansine, or T-DM1. A decrease in LVEF was seen in $1.2 \%$ of patients receiving adjuvant T-DM1 $1.7 \%$ of patients receiving T-DM1 in the advanced setting EMILIA trials [31, 32]. Of note, within both clinical trials,

Table 2 FDA indication for HER2-targeted agents in breast cancer

\begin{tabular}{ll}
\hline Targeted agent & Clinical indication \\
\hline Trastuzumab [25, 26] & - Adjuvant treatment for HER2 + breast cancer as part of a treatment regimen that includes doxorubicin, cyclo- \\
phosphamide, and paclitaxel or docetaxel OR part of a treatment regimen with docetaxel and carboplatin OR \\
single agent following anthracycline-based therapy \\
- Metastatic HER2 + breast cancer in combination with trastuzumab and docetaxel for first-line treatment \\
- Adjuvant treatment of early stage HER2 + breast cancer at high risk of recurrence in combination with trastu- \\
Pertuzumab [27] \\
- Neoadjuvant treatment of locally advanced, inflammatory or early stage HER2 + breast cancer in combination \\
with trastuzumab and chemotherapy \\
- Advanced or metastatic HER2 + breast cancer in combination with capecitabine after prior therapy including \\
an anthracycline, a taxane, and Trastuzumab \\
- Metastatic HER2 +, hormone-receptor positive breast cancer in postmenopausal women in combination with \\
letrozole \\
- Extended adjuvant treatment of early-stage HER2 + breast cancer after adjuvant trastuzumab-based therapy \\
- Advanced/metastatic HER2 + breast cancer in combination with capecitabine after 2 prior anti-HER2 based \\
regimens in metastatic setting \\
- Unresectable/metastatic HER2 + breast cancer in combination with Trastuzumab and capecitabine after 1 prior \\
anti-HER2 based regimen \\
- Treatment of early-stage HER2 + breast cancer with residual invasive-disease following neoadjuvant taxane \\
and trastuzumab-based treatment \\
- Metastatic HER2 + breast cancer after previous trastuzumab and taxane therapy \\
- Metastatic HER2 + breast cancer after 2 prior lines of HER2-based regimens in metastatic setting
\end{tabular}

Abbreviations: HER2 +, Human Epidermal Growth Factor Receptor 2 Positive; $L V E F$, left ventricular ejection fraction; T-DMI, Trastuzumab Emtansine 
Fig. 1 Mechanism of action for HER2-targeted agents. Created with BioRender.com

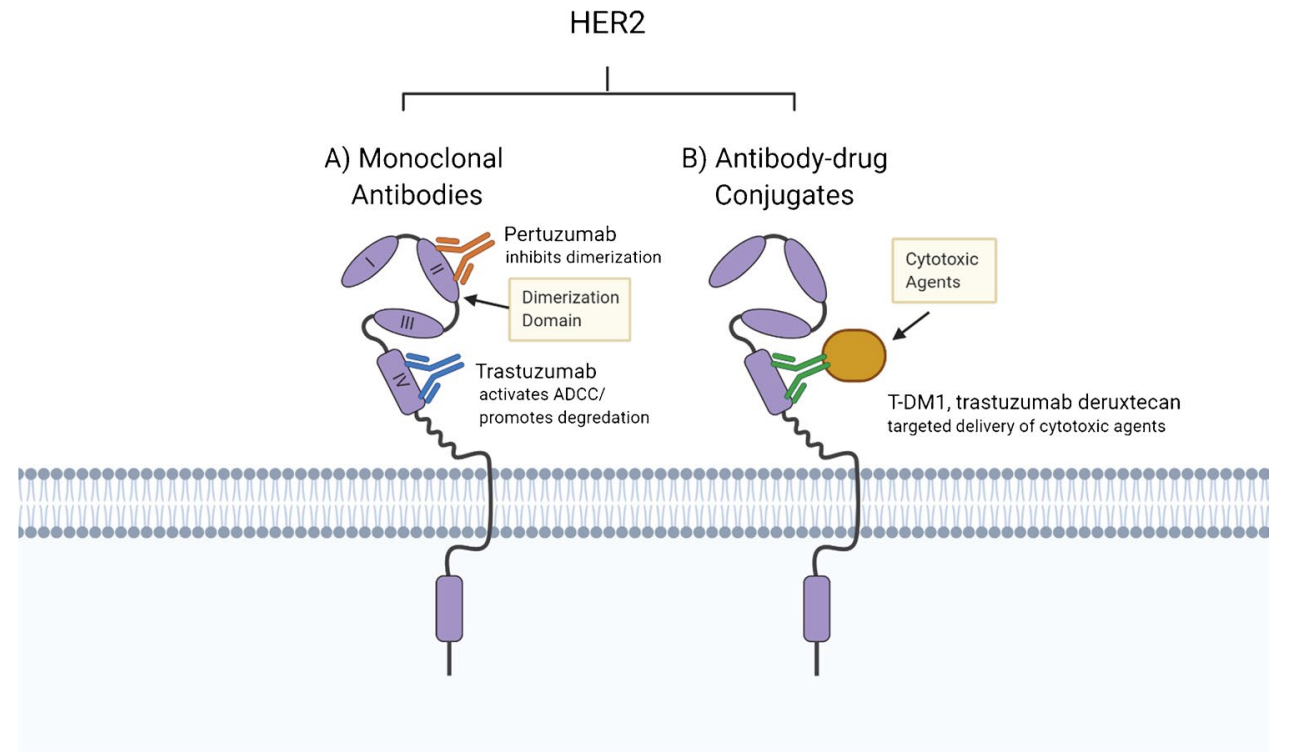

C) Tyrosine Kinase Inhibitors

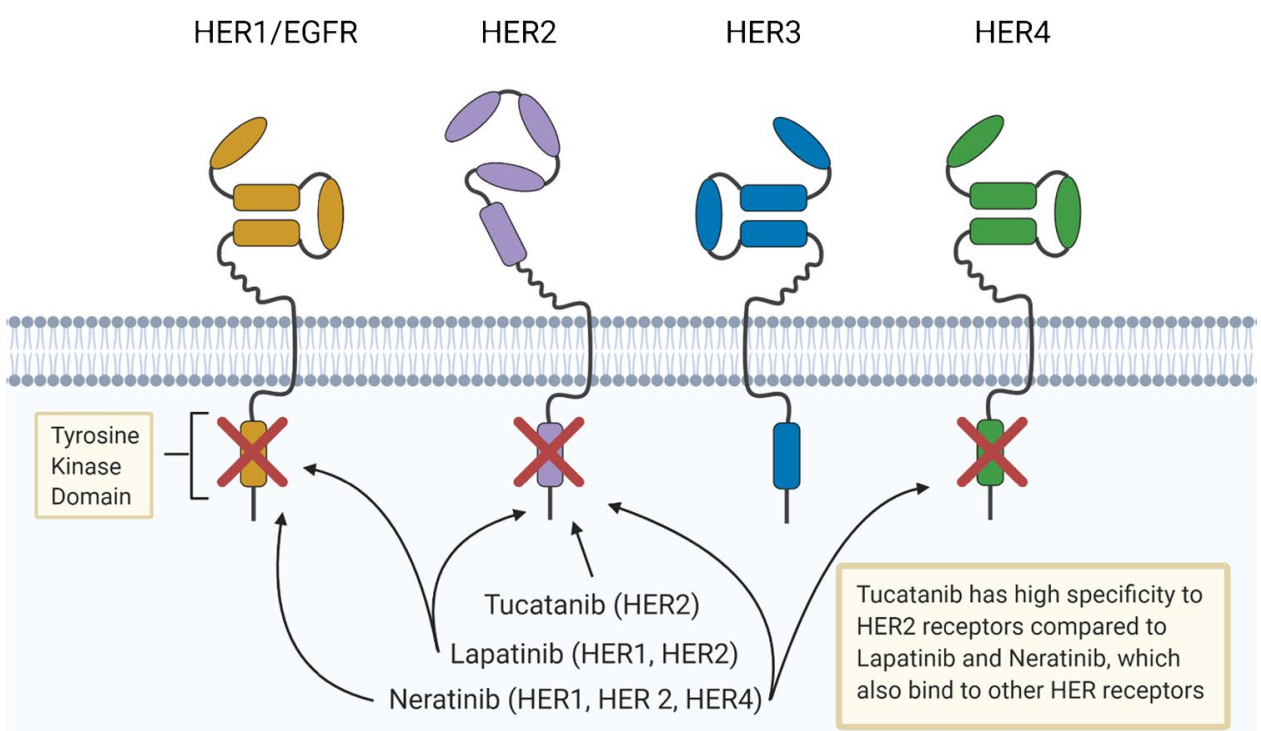

patients had to have an LVEF of greater than or equal to $50 \%$ to be eligible for the study. Therefore, cardiotoxicity rates reported in these trials may not be fully reflective of patients within the community setting.

\section{Tyrosine Kinase Inhibitors}

Several oral HER2-targeted tyrosine kinase inhibitors (TKIs) are currently available for patients with HER2-positive breast cancer, including lapatinib, neratinib, and tucatinib (Fig. 1) Lapatinib, which targets HER1 and HER2 and is approved in the advanced setting for HER2-positive breast cancer, was initially approved by the FDA in 2007, followed by neratinib and tucatinib in 2017 and 2020, respectively.
Although less frequent than trastuzumab, lapatinib has been associated with an increased risk of cardiac events, including decreased LVEF and QTc prolongation requiring the need for cardiac monitoring and dose modifications [33, 34]. ALTERNATIVE, a phase III trial, randomized patients with advanced breast cancer to lapatinib (L) in combination with an aromatase inhibitor (AI) with or without trastuzumab $(\mathrm{H})$ versus $\mathrm{H}$ and an AI (LAI, LHAI, or HAI). As a secondary endpoint, investigators assessed patients for cardiac safetyrelated events. They found that $7 \%$ of patients receiving LHAI experienced a cardiac event compared to only $3 \%$ of patients receiving HAI and $2 \%$ of patients receiving LAI. However, the study was not powered to detect a statistical difference [20]. In contrast, a subgroup analysis of the 
ALTTO trial, comparing adjuvant treatment of trastuzumab with or without lapatinib found that patients receiving combination therapy seemed to have a compensation for cardiac events primarily during the first 6 months of trastuzumab use, as evidenced by a trend towards a higher cumulative incidence of cardiac events in the trastuzumab-only arm after 6 months [12]. The proposed mechanism of cardiac safety seen with lapatinib may be explained by the activation of the AMPK pathway, which inhibits TNF-alpha-induced cardiomyocyte cell death, as opposed to trastuzumab, which inhibits the AMPK pathway [35]. Lower rates of cardiotoxicity may also be related to selective inclusion and exclusion criteria. These findings suggest that neratinib and tucatinib may potentially confer some degree of cardiac risk.

Neratinib is an irreversible, pan-HER TKI that inhibits HER 1,2, and 4 and is approved as monotherapy for extended adjuvant treatment after trastuzumab-based therapy and for use in advanced HER2-positive breast cancer in combination with capecitabine [29]. The ExteNET trial led to the FDA approval of neratinib for extended adjuvant treatment (after 1 year of trastuzumab) [36]. Compared to placebo, 1 year of neratinib therapy demonstrated an improvement in 2-year invasive disease-free survival, particularly in those patients with hormone-receptor-positive disease and positive lymph nodes. These findings were sustained at 5 years [36]. Additionally, the use of neratinib was not associated with an increase in long-term toxicity, specifically cardiotoxicity, although patients with clinically significant cardiac morbidities were excluded [36]. Similarly in the NALA trial, neratinib was compared to lapatinib, both in combination with capecitabine, and no significant cardiotoxicity concerns were noted. Patients receiving neratinib experienced similar rates of arrhythmia and ischemic heart disease, as well as lower rates of QTc prolongation. However, there was a slightly higher incidence of LVEF reduction in the neratinib-arm [21]. No grade 5 cardiac events were seen in the neratinib arm, compared to two in the lapatinib arm (i.e., cardiac arrest, cardiac tamponade) [21]. However, when compared to lapatinib on a mechanistic level, neratinib targets an additional receptor, HER4 [29]. Both HER2 and HER4 are expressed on cardiomyocytes, and the activation of HER2/ HER4 heterodimers may be essential in maintaining ventricular structure, function, and overall cell survival [37, 38].

Tucatinib selectively targets HER2 and is approved for use in advanced HER2-positive breast cancer [39]. In an early phase I study investigating tucatinib in heavily pretreated patients with HER2-positive solid tumors, no cardiac-related symptoms or side effects were identified [40]. Notably, all patients had received prior trastuzumab, and $84 \%$ had received prior lapatinib [40]. Patients were also excluded if their LVEF was less than 40\% [40]. Similarly, in an unpublished study evaluating the effect of tucatinib $300 \mathrm{mg}$ daily on cardiac repolarization in healthy subjects, no effect on QT prolongation was noted (NCT03777761) [41]. More recently, the phase III HER2CLIMB study investigated tucatinib vs. placebo in combination with trastuzumab and capecitabine in patients who had received prior trastuzumab and ado-trastuzumab emtansine [22]. This combination was associated with a significant improvement in progression-free survival, including patients with active brain metastases [22, 23]. The incidence of all-grade, as well as grade $>3$ treatment-related adverse events, was similar between arms, specifically in regards to LVEF reduction and QTc prolongation [23]. A total of 14 subjects experienced treatment-related LVEF reduction, with nine patients $(2.2 \%)$ in the tucatinib arm and five patients $(2.5 \%)$ in the placebo arm. Additionally, two patients in each arm experienced a grade 5 cardiac adverse event [23]. Similar rates of discontinuation and dose modification were also seen. As the role of HER3 in cardiac function is less established, and this medication was only recently approved, our understanding of drug-related cardiotoxicity is relatively limited [42].

One of the limitations of clinical trials with HER2-targeted therapies is the exclusion of patients with a cardiac history or reduced LVEF at baseline before therapy initiation. Because of these exclusion criteria, cardiotoxicity rates seen within the clinical trial setting are likely not reflective of patients treated in the community. There is little guidance on managing patients that may have reduced LVEF at baseline or previous cardiac history. Recent data from a small prospective study, SAFE-HEaRt, demonstrated that patients with stage I-IV HER2-positive breast cancer receiving trastuzumab, pertuzumab, or T-DM1 with an LVEF 40-49\%, without heart failure symptoms, were able to complete their planned HER2-targeted therapies with appropriate guided medical therapy [43]. This data is promising and supports the consideration of HER2-targeted agents in patients otherwise excluded from clinical trials. Additional clinical trials are needed in patients with cardiac risk factors and/ or cardiac disease and reduced LVEF to determine toxicity rates more reflective of those likely seen within the community setting as well as to provide safety data for treatment. SCHOLAR-2, a prospective randomized trial, will evaluate whether it is safe and effective to continue trastuzumab, pertuzumab, or TDM-1 in patients with early stage HER2 positive breast cancer who develop mild, minimally symptomatic or asymptomatic systolic left ventricular dysfunction (NCT04680442).

\section{Cardiovascular Monitoring Strategies for HER2-Targeted Therapy}

Cardiovascular monitoring of patients on HER2-targeted therapy has primarily been based on studies with trastuzumab alone [25]. The FDA recommends a cardiac 
assessment at baseline that includes a history and physical examination, and evaluation of LVEF by echocardiogram or MUGA scan, and every three months during and every 6 months following completion of trastuzumab as a component of adjuvant therapy for a maximum of two years regardless of anthracycline use (see Fig. 2A) [25]. Similar cardiac monitoring strategies have been recommended by the European Society for Medical Oncology (ESMO) [44, 45], American Society of Clinical Oncology (ASCO) [46], and National Comprehensive Cancer Network (NCCN) [47]. Cardiac monitoring in the setting of dual HER2 blockade as per the product monograph recommends LVEF assessment prior to initiation of pertuzumab (in combination with trastuzumab) and at regular intervals (e.g., every three months) during treatment to ensure LVEF remains within the institution's normal limits. The product monograph does not specifically address cardiac monitoring upon completing pertuzumab when given in the adjuvant setting. There are no specific guidelines regarding cardiac monitoring with
HER2 therapy in the advanced breast cancer setting. This is particularly relevant given that individuals may remain on HER2 therapy for months to years. In a population-based study in Ontario, where cardiac monitoring is required every 6 months for advanced breast cancer, the incidence of cardiotoxicity was low [48]. In light of the established clinical benefit of sequential HER2-targeted therapy in advanced breast cancer, we recommend routine cardiac monitoring only in the setting of clinical heart failure or for those patients with significant cardiovascular risk factors or preexisting cardiomyopathy in order to optimize cardioprotective strategies. Routine cardiac monitoring in asymptomatic patients may lead to early termination of life-sustaining HER2-targeted therapy.

There are several drawbacks to the current cardiac monitoring algorithms. First, inter-user variability is possible between echocardiography-based LVEF measurement $(+/-10 \%)$, leading to unnecessary further cardiovascular testing and discontinuation of lifesaving cancer therapy
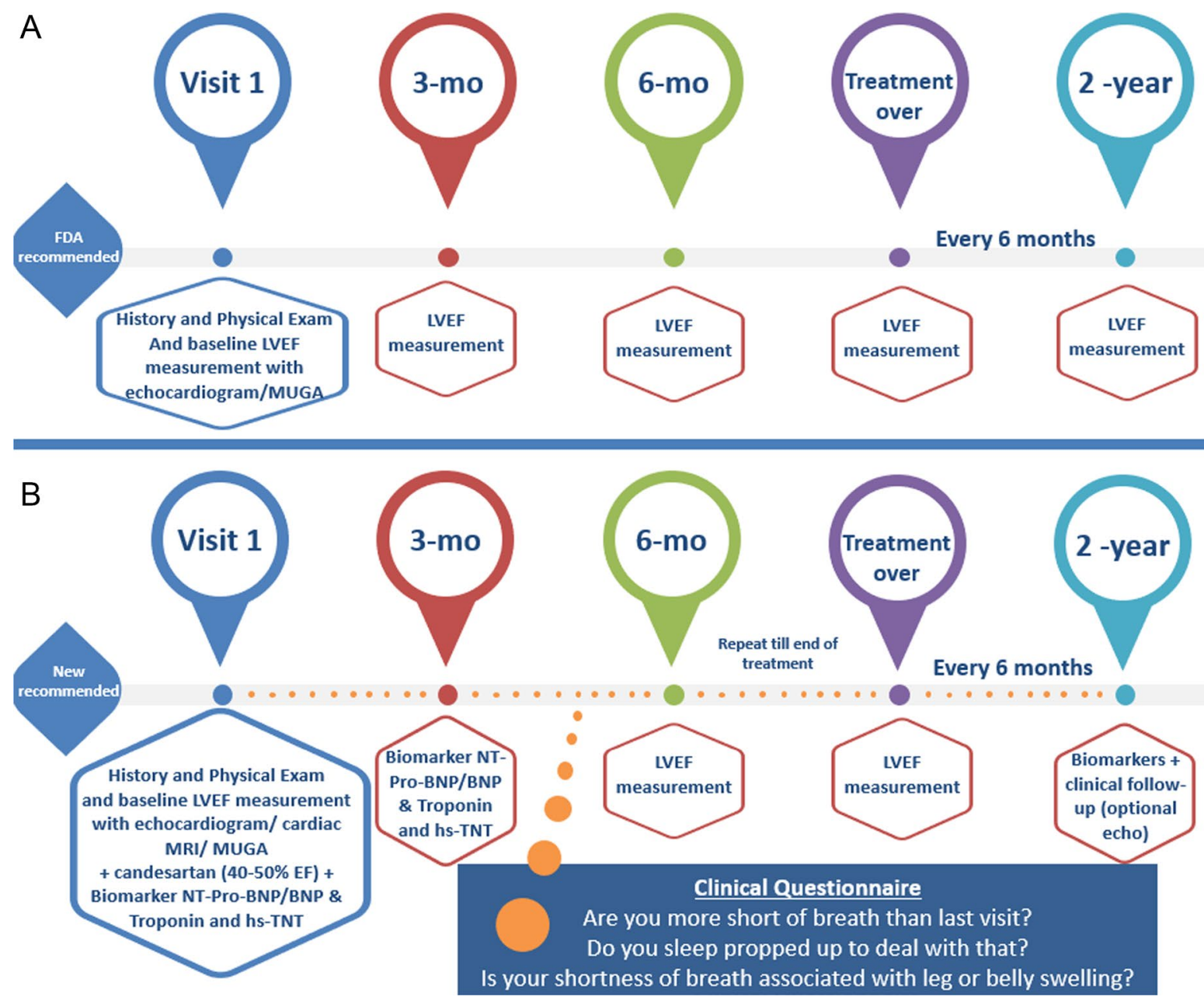

Fig. 2 A-Current recommended cardiac monitoring for early-stage breast cancer patients on HER2-targeted therapy. B-Suggested cardiac monitoring for early-stage breast cancer patients on HER2-targeted therapy 
[49]. In the light of findings from the recently published SUCCOUR trial, there may be a benefit to high-quality echocardiographic strain imaging; however, it is essential to note that the majority of patients treated with HER2targeted therapy will not experience clinically significant cardiotoxicity and, therefore, may not need such intense cardiovascular imaging [7, 50]. In the era of COVID-19, where efforts are being made to minimize patient contact, the current recommendations for cardiovascular monitoring seem excessive (Fig. 2A) [51]. ESMO recently published a position statement on COVID-19 and cardiovascular imaging that ranks the first echocardiogram in early-stage breast cancer patients a medium priority and follow-up echocardiograms as low-priority tests in the absence of symptoms [52]. Finally, unnecessary tests in cancer treatment settings add to patients' emotional and financial burden $[52,53]$. Studies have demonstrated in the USA that only $46 \%$ of women below the age of 65 [54] and 36\% of those above the age of 65 [55] have cardiac monitoring as per the FDA recommendations.

In Fig. 2B, we propose an alternate pathway of monitoring breast cancer patients who are receiving HER2-targeted therapy. All patients should have a history and physical and a baseline echocardiogram/cardiac MRI/MUGA scan based on institutional availability. The initial evaluation of patients should consider active management of modifiable cardiovascular risk factors, especially hypertension (except non-dihydropyridine calcium channel blockers due to interaction leading to anti-HER2 level rise), encouraging exercise, and a healthy diet $[45,56,57]$. N-terminal pro-brain natriuretic peptide (NT-pro-BNP)/ brain-natriuretic peptide (BNP) is a highly sensitive marker of myocardial stretch, which increases with an increase in volume status, which can be seen in those with clinical heart failure. Troponin-T (TnT)/high-sensitivity troponin (hs-TnT) is a highly sensitive marker of myocardial necrosis, thus useful as a marker of early cardiotoxicity. Baseline cardiac biomarkers can be drawn, with cancer treatment bloodwork, prior to initiation of HER2-targeted therapy. A clinical questionnaire regarding signs and symptoms of heart failure can be administered during cancer clinic visits. This would provide an alternate standardized way of evaluating patients for potential cardiovascular toxicity without additional clinical visits or cardiac imaging. At the prescribed 3-month time point, cardiac biomarkers can be drawn before the scheduled anti-HER2 infusion, omitting the need for an echocardiogram, if normal. This cardiovascular monitoring strategy would minimize contact while potentially providing the same level of drugtoxicity monitoring.

A low-dose angiotensin-receptor blocker (e.g., candesar$\tan$ ), ACE inhibitor, and/or beta-blocker should be considered prior to initiation of or during HER2-targeted therapy in patients with LV dysfunction (LVEF 40-50\%). SCHOLAR [58] and SAFE-HEaRT [43] were small prospective studies demonstrating the feasibility and safety of continuing with trastuzumab therapy in the setting of mild to moderate left ventricular dysfunction (i.e., LVEF 40-49\%) with the use of ACE inhibitors and/or beta-blockers. In a recent populationbased study (5500 women) in Ontario, patients who did not complete the recommended course of adjuvant trastuzumab, including those with cardiotoxicity, had a statistically higher risk of breast cancer relapse and death [59]. A recent study from Memorial Sloan Kettering Cancer Center (1396 women) corroborated an increased risk of breast cancer recurrence and death with interruption of trastuzumab therapy, 24\% (interrupted) vs. 13\% (continuous), highlighting

Table 3 Primary cardiotoxicity prevention trials in patients with breast cancer [61]

\begin{tabular}{|c|c|c|c|}
\hline Trial $(N)$ & Intervention & Primary outcome & Benefit (yes/no) \\
\hline $\begin{array}{l}\quad \text { PRADA }[63] \\
(N=130, \text { all anthracycline, } 22 \% \\
\text { trastuzumab })\end{array}$ & $\begin{array}{l}\text { 1:1:1:1, metoprolol, candesartan, } \\
\text { metoprolol and candesartan, or } \\
\text { placebo }\end{array}$ & $\begin{array}{l}\text { Changes in LVEF by CMR } \\
\text { at } 10 \text { to } 64 \text { weeks }\end{array}$ & $\begin{array}{l}\text { Yes, absolute LVEF change: } 2.6 \% \text { in } \\
\text { placebo, } 0.8 \% \text { in candesartan }(p=0.026)\end{array}$ \\
\hline $\begin{array}{l}\text { Guglin et al. }[64] \\
(N=468, \text { all trastuzumab, } 40 \% \\
\text { doxorubicin })\end{array}$ & $\begin{array}{l}\text { 1:1:1 carvedilol, lisinopril, or } \\
\text { placebo }\end{array}$ & $\begin{array}{l}\text { LVEF }>10 \% \text { or LVEF } \\
\text { decline }>5 \% \text { with absolute } \\
\text { LVEF }<50 \%\end{array}$ & $\begin{array}{l}\text { Yes, }>10 \% \text { LVEF decline in subset } \\
\text { with prior anthracycline exposure: } 47 \% \\
\text { placebo, } 31 \% \text { carvedilol, } 37 \% \text { lisinopril } \\
(p=0.009)\end{array}$ \\
\hline $\begin{array}{l}\text { Boekhout et al. [65] } \\
(N=206 \text {, all epirubicin with tras- } \\
\text { tuzumab) }\end{array}$ & 1:1 candesartan or placebo & $\begin{array}{l}\text { LVEF decline of }>15 \% \text { or a } \\
\text { decrease below the abso- } \\
\text { lute value of } 45 \%\end{array}$ & $\begin{array}{l}\text { No, LVEF decline: } 19 \% \text { in candesartan, } \\
16 \% \text { in placebo }(p=0.58)\end{array}$ \\
\hline $\begin{array}{l}\text { MANTICORE[66] } \\
(N=94, \text { all trastuzumab, } 12-33 \% \\
\text { anthracycline })\end{array}$ & $\begin{array}{l}\text { 1:1:1 bisoprolol, perindopril, or } \\
\text { placebo }\end{array}$ & $\begin{array}{l}\text { Changes in LVEDVI by } \\
\text { CMR at } 1 \text { year }\end{array}$ & $\begin{array}{l}\text { Yes, Small reduction in LVEF decline } \\
\text { with bisoprolol compared with perindo- } \\
\text { pril and placebo (-1\% vs. }-3 \% \text { vs. }-5 \% \text {, } \\
p=0.001)\end{array}$ \\
\hline
\end{tabular}

Abbreviations: $C M R$, cardiac magnetic resonance imaging; $L V E D V I$, left ventricular end-diastolic volume index; $L V E F$, left ventricular ejection fraction; MANTICORE, Multidisciplinary Approach to Novel Therapies in Cardio-oncology Research; PRADA, Prevention of Cardiac Dysfunction During Adjuvant Breast Cancer Therapy 
the importance of continued investigation of monitoring, prevention, and management of cardiac dysfunction to limit treatment interruption or cessation [60]. Several prospective studies, namely, PRADA, MANTICORE, Guglin et al., Boekhout et al., suggest ACE inhibitors and/or beta-blockers for primary prevention in HER2-positive breast cancer patients with/without combination anthracycline therapy (Table 3) [61]. While these strategies may facilitate patients completing a recommended course of HER2-targeted therapy, larger prospective studies in "at risk" (e.g., low LVEF of $50 \%$ at baseline) populations are needed prior to widespread adoption of this approach in clinical practice. A recent metaanalysis of 17 trials of neurohormonal therapies in cancer patients exposed to potentially cardiotoxic cancer therapy, noted higher left ventricular ejection fractions (although absolute changes were small), compared to placebo; however, significant heterogeneity was observed highlighting the need for larger prospective studies [62].

In summary, all patients offered HER2-targeted therapy should have baseline cardiac assessment that includes a history and physical examination, and cardiac imaging. We recommend replacing 3-, 9-, and 15-month cardiac monitoring in asymptomatic individuals, with a short cardiovascular questionnaire at each infusion visit, along with the addition of cardiac biomarkers.

\section{Conclusion}

HER2-targeted therapies have led to significant improvements in clinical outcomes for patients with early and advanced HER2-positive breast cancer. The pivotal clinical trials reported an increased risk of heart failure (up to 5\%) in patients with HER2-positive breast cancer treated with trastuzumab; however, long-term follow-up $(10+$ years $)$ of these studies has failed to demonstrate increased rates of cardiotoxicity over time, attesting to the long-term safety of this class of drugs for the majority of patients. Several novel HER2-targeted therapies have been approved by the FDA in the last few years, particularly for patients with advanced disease. Clinicians should be reassured that to date, there is no indication that these agents are associated with a greater risk of cardiotoxicity, although there is less experience with these drugs in the non-clinical trial patient population. Intense cardiac monitoring is currently recommended for all patients during HER2-targeted treatment, irrespective of pre-existing cardiovascular risk or co-morbidities. We propose an alternative cardiac monitoring strategy that incorporates patients' baseline cardiovascular risk, cardiac symptoms, cardiac imaging, and biomarkers for patients treated with HER2-targeted therapies.

\section{Declarations}

Conflict of Interest Susan F. Dent has received compensation from Seattle Genetics for participating on advisory boards. Amber Morse declares that she has no conflict of interest. Sarah E Burnette declares that she has no conflict of interest. Avirup Guha declares that he has no conflict of interest. Heather Moore has received compensation from AstraZeneca/Daiichi-Sankyo and Seattle Genetics for participating on advisory boards.

\section{References}

1. Cameron D, Piccart-Gebhart MJ, Gelber RD, Procter M, Goldhirsch A, de Azambuja E, et al. 11 years' follow-up of trastuzumab after adjuvant chemotherapy in HER2-positive early breast cancer: final analysis of the HERceptin Adjuvant (HERA) trial. Lancet. 2017;389(10075):1195-205.

2. Perez EA, Romond EH, Suman VJ, Jeong J-H, Davidson NE, Geyer CE Jr, et al. Four-year follow-up of trastuzumab plus adjuvant chemotherapy for operable human epidermal growth factor receptor 2-positive breast cancer: joint analysis of data from NCCTG N9831 and NSABP B-31. J Clin Oncol. 2011;29(25):3366.

3. Slamon D, Eiermann W, Robert N, Pienkowski T, Martin M, Press $\mathrm{M}$, et al. Adjuvant trastuzumab in HER2-positive breast cancer. N Engl J Med. 2011;365(14):1273-83.

4. Thavendiranathan P, Abdel-Qadir H, Fischer HD, Camacho X, Amir E, Austin PC, et al. Breast cancer therapy-related cardiac dysfunction in adult women treated in routine clinical practice: a population-based cohort study. J Clin Oncol. 2016;34(19):2239-46.

5. Lidbrink E, Chmielowska E, Otremba B, Bouhlel A, Lauer S, Hermoso ML, et al. A real-world study of cardiac events in> 3700 patients with HER2-positive early breast cancer treated with trastuzumab: final analysis of the OHERA study. Breast Cancer Res Treat. 2019;174(1):187-96.

6. Banke A, Fosbøl EL, Ewertz M, Videbæk L, Dahl JS, Poulsen $\mathrm{MK}$, et al. Long-term risk of heart failure in breast cancer patients after adjuvant chemotherapy with or without trastuzumab. JACC Heart Fail. 2019;7(3):217-24.

7. Swain SM, Kim S-B, Cortés J, Ro J, Semiglazov V, Campone M, et al. Pertuzumab, trastuzumab, and docetaxel for HER2-positive metastatic breast cancer (CLEOPATRA study): overall survival results from a randomised, double-blind, placebo-controlled, phase 3 study. Lancet Oncol. 2013;14(6):461-71.

8. Bowles EJA, Wellman R, Feigelson HS, Onitilo AA, Freedman AN, Delate T, et al. Risk of heart failure in breast cancer patients after anthracycline and trastuzumab treatment: a retrospective cohort study. J Natl Cancer Inst. 2012;104(17):1293-305.

9. Slamon DJ, Eiermann W, Robert NJ, Giermek J, Martin M, Jasiówka M, et al. Abstract S5-04: Ten year follow-up of BCIRG006 comparing doxorubicin plus cyclophosphamide followed by docetaxel $(\mathrm{AC} \rightarrow \mathrm{T})$ with doxorubicin plus cyclophosphamide followed by docetaxel and trastuzumab $(\mathrm{AC} \rightarrow \mathrm{TH})$ with docetaxel, carboplatin and trastuzumab (TCH) in HER2+ early breast cancer. Can Res. 2016;76:S5-04.

10. Von Minckwitz G, Procter M, de Azambuja E, Zardavas D, Benyunes M, Viale G, et al. Adjuvant pertuzumab and trastuzumab in early HER2-positive breast cancer. N Engl J Med. 2017;377(2):122-31.

11. Piccart M PM FD, Azambuja E, Clark E, Ewer M, Restuccia E, Jerusalem G, Dent S, Reaby L, Bonnefoi H, Krop I, Liu T, Pieńkowski T, Toi M, Wilcken N, Andersson M, Im Y, Tseng 
L, Lueck H, Colleoni M. Monturus E, Sicoe M, Guillaume S, Bines J, Gelber R, Viale G and Thomssen C. Updated APHINITY trial data show addition of pertuzumab to trastuzumab plus chemotherapy continues to yield clinical benefit in patients with operable HER2-positive early breast cancer [abstract]. . 2019 San Antonio Breast Cancer Symposium (SABCS). December 11, 2019;GS1-04.

12. Eiger D, Pondé NF, Agbor-Tarh D, Moreno-Aspitia A, Piccart M, Hilbers FS, et al. Long-term cardiac outcomes of patients with HER2-positive breast cancer treated in the adjuvant lapatinib and/or trastuzumab treatment optimization trial. Br J Cancer. 2020;122(10):1453-60.

13. Lambertini M, Campbell C, Gelber RD, Viale G, McCullough A, Hilbers F, et al. Dissecting the effect of hormone receptor status in patients with HER2-positive early breast cancer: exploratory analysis from the ALTTO (BIG 2-06) randomized clinical trial. Breast Cancer Res Treat. 2019;177(1):103-14.

14. Chan A, Moy B, Mansi J, Ejlertsen B, Holmes FA, Chia S, et al. Final efficacy results of neratinib in HER2-positive hormone receptor-positive early-stage breast cancer from the phase III ExteNET trial. Clin Breast Cancer. 2021;21(1):80-91. e7.

15. Chan A, Delaloge S, Holmes FA, Moy B, Iwata H, Harvey VJ, et al. Neratinib after trastuzumab-based adjuvant therapy in patients with HER2-positive breast cancer (ExteNET): a multicentre, randomised, double-blind, placebo-controlled, phase 3 trial. Lancet Oncol. 2016;17(3):367-77.

16. Swain SM, Miles D, Kim S-B, Im Y-H, Im S-A, Semiglazov V, et al. Pertuzumab, trastuzumab, and docetaxel for HER2-positive metastatic breast cancer (CLEOPATRA): end-of-study results from a double-blind, randomised, placebo-controlled, phase 3 study. Lancet Oncol. 2020;21(4):519-30.

17. Diéras V, Miles D, Verma S, Pegram M, Welslau M, Baselga J, et al. Trastuzumab emtansine versus capecitabine plus lapatinib in patients with previously treated HER2-positive advanced breast cancer (EMILIA): a descriptive analysis of final overall survival results from a randomised, open-label, phase 3 trial. Lancet Oncol. 2017;18(6):732-42.

18. Modi S, Saura C, Yamashita T, Park YH, Kim S-B, Tamura K, et al. Trastuzumab deruxtecan in previously treated HER2-positive breast cancer. N Engl J Med. 2020;382(7):610-21.

19. Modi S, Park H, Murthy RK, Iwata H, Tamura K, Tsurutani J, et al. Antitumor activity and safety of trastuzumab deruxtecan in patients with HER2-low-expressing advanced breast cancer: results from a phase Ib study. J Clin Oncol: Off J Am Soc Clin Oncol. 2020;38(17):1887-96.

20. Johnston SR, Hegg R, Im S-A, Park IH, Burdaeva O, Kurteva $\mathrm{G}$, et al. Phase III, randomized study of dual human epidermal growth factor receptor 2 (HER2) blockade with lapatinib plus trastuzumab in combination with an aromatase inhibitor in postmenopausal women with HER2-positive, hormone receptorpositive metastatic breast cancer: ALTERNATIVE. J Clin Oncol. 2018;36(8):741.

21. Saura C, Oliveira M, Feng Y-H, Dai M-S, Chen S-W, Hurvitz SA, et al. Neratinib plus capecitabine versus lapatinib plus capecitabine in HER2-positive metastatic breast cancer previously treated with $\geq 2$ HER2-directed regimens: phase III NALA trial. J Clin Oncol. 2020;38(27):3138

22. Lin NU, Borges V, Anders C, Murthy RK, Paplomata E, Hamilton $\mathrm{E}$, et al., editors. Intracranial efficacy and survival with tucatinib plus trastuzumab and capecitabine for previously treated HER2positive breast cancer with brain metastases in the HER2CLIMB trial2020: American Society of Clinical Oncology.

23. Murthy RK, Loi S, Okines A, Paplomata E, Hamilton E, Hurvitz SA, et al. Tucatinib, trastuzumab, and capecitabine for HER2-positive metastatic breast cancer. N Engl J Med. 2020;382(7):597-609.
24. Serrano C, Cortes J, De Mattos-Arruda L, Bellet M, Gomez $\mathrm{P}$, Saura $\mathrm{C}$, et al. Trastuzumab-related cardiotoxicity in the elderly: a role for cardiovascular risk factors. Ann Oncol. 2012;23(4):897-902.

25. FDA. HERCEPTIN® (trastuzumab) 2010 [updated 10/2010].

26. HERCEPTIN (trastuzumab) [package insert]. South San Francisco: Genentech I.

27. Perjeta (Pertuzumab) [prescribing information]. South San Francisco, 2012.

28. TYKERB (lapatinib) [package insert]. Reserach Triangle Park NG.

29. Nerlynx (neratinib) [prescribing information]. Los Angeles CPB, Inc; 2020.

30. Fam-trastuzumab deruxtecan [package insert]. Basking Ridge: Daiichi Sankyo. 2019.

31. Von Minckwitz G, Huang C-S, Mano MS, Loibl S, Mamounas EP, Untch M, et al. Trastuzumab emtansine for residual invasive HER2-positive breast cancer. N Engl J Med. 2019;380(7):617-28.

32. Verma S, Miles D, Gianni L, Krop IE, Welslau M, Baselga J, et al. Trastuzumab emtansine for HER2-positive advanced breast cancer. N Engl J Med. 2012;367(19):1783-91.

33. Perez EA, Koehler M, Byrne J, Preston AJ, Rappold E, Ewer MS, editors. Cardiac safety of lapatinib: pooled analysis of 3689 patients enrolled in clinical trials. Mayo Clinic Proceedings; 2008: Elsevier.

34. Sendur MA, Aksoy S, Altundag K. Cardiotoxicity of novel HER2targeted therapies. Curr Med Res Opin. 2013;29(8):1015-24.

35. Shell SA, Lyass L, Trusk PB, Pry KJ, Wappel RL, Bacus SS. Activation of AMPK is necessary for killing cancer cells and sparing cardiac cells. Cell Cycle. 2008;7(12):1769-75.

36. Martin M, Holmes FA, Ejlertsen B, Delaloge S, Moy B, Iwata $\mathrm{H}$, et al. Neratinib after trastuzumab-based adjuvant therapy in HER2-positive breast cancer (ExteNET): 5-year analysis of a randomised, double-blind, placebo-controlled, phase 3 trial. Lancet Oncol. 2017;18(12):1688-700.

37. Albini A, Cesana E, Donatelli F, Cammarota R, Bucci EO, Baravelli $\mathrm{M}$, et al. Cardio-oncology in targeting the HER receptor family: the puzzle of different cardiotoxicities of HER2 inhibitors. Future Cardiol. 2011;7(5):693-704.

38. Rochais F, Fischmeister R. Acute cardiac effects of neuregulin-1/ ErbB signalling. Cardiovasc Res. 2010;88(3):393-4.

39. Tukysa (tucatinib) [prescribing information]. Bothell WSGIA.

40. Moulder SL, Borges VF, Baetz T, Mcspadden T, Fernetich G, Murthy RK, et al. Phase I study of ONT-380, a HER2 inhibitor, in patients with HER2+-advanced solid tumors, with an expansion cohort in HER2+ metastatic breast cancer (MBC). Clin Cancer Res. 2017;23(14):3529-36.

41. ClinicalTrials.gov [Internet]. Identifier NCT03777761, effects of tucatinib on cardiac repolarization in healthy participants. 2018 Dec 17 [cited 2020 Dec 1].

42. De Keulenaer GW, Feyen E, Dugaucquier L, Shakeri H, Shchendrygina A, Belenkov YN, et al. Mechanisms of the multitasking endothelial protein NRG-1 as a compensatory factor during chronic heart failure. Circ Heart Fail. 2019;12(10):e006288.

43. Lynce F, Barac A, Geng X, Dang C, Yu A, Smith K, et al. Prospective evaluation of the cardiac safety of HER2-targeted therapies in patients with HER2-positive breast cancer and compromised heart function: the SAFE-HEaRt study. Breast Cancer Res Treat. 2019;175(3):595-603.

44. Cardoso F, Kyriakides S, Ohno S, Penault-Llorca F, Poortmans P, Rubio I, et al. Early breast cancer: ESMO Clinical Practice Guidelines for diagnosis, treatment and follow-up. Ann Oncol. 2019;30(8):1194-220.

45. Curigliano G, Lenihan D, Fradley M, Ganatra S, Barac A, Blaes A, et al. Management of cardiac disease in cancer patients 
throughout oncological treatment: ESMO consensus recommendations. Ann Oncol. 2020;31(2):171-90.

46. Giordano SH, Temin S, Kirshner JJ, Chandarlapaty S, Crews JR, Davidson NE, et al. Systemic therapy for patients with advanced human epidermal growth factor receptor 2-positive breast cancer: American Society of Clinical Oncology clinical practice guideline. J Clin Oncol. 2014;32(19):2078.

47. Gradishar WJ, Anderson BO, Abraham J, Aft R, Agnese D, Allison KH, et al. Breast cancer, version 3.2020, NCCN clinical practice guidelines in oncology. J Natl Compr Canc Netw. 2020;18(4):452-78.

48. Rushton-Marovac MK, Lima I, Tuna M, Melloni C, Pritchard K, Hawken S, et al. Abstract P5-14-11: Cardiac monitoring in advanced breast cancer patients treated with trastuzumab: does it improve cardiac safety? Cancer Res. 2020;80(4 Supplement):P5-14-1-P5--1.

49. Thavendiranathan P, Grant AD, Negishi T, Plana JC, Popović ZB, Marwick TH. Reproducibility of echocardiographic techniques for sequential assessment of left ventricular ejection fraction and volumes: application to patients undergoing cancer chemotherapy. J Am Coll Cardiol. 2013;61(1):77-84.

50. Schneeweiss A, Chia S, Hickish T, Harvey V, Eniu A, Hegg R, et al. Pertuzumab plus trastuzumab in combination with standard neoadjuvant anthracycline-containing and anthracycline-free chemotherapy regimens in patients with HER2-positive early breast cancer: a randomized phase II cardiac safety study (TRYPHAENA). Ann Oncol. 2013;24(9):2278-84.

51. Addison D, Campbell CM, Guha A, Ghosh AK, Dent SF, Jneid H. Cardio-oncology in the era of the COVID-19 pandemic and beyond. J Am Heart Assoc. 2020;9(19):e017787.

52. Cheng AC, Levy MA, editors. Determining burden of commuting for treatment using online mapping services-a study of breast cancer patients. AMIA Annual Symposium Proceedings; 2017: American Medical Informatics Association.

53. Bubis LD, Davis L, Mahar A, Barbera L, Li Q, Moody L, et al. Symptom burden in the first year after cancer diagnosis: an analysis of patient-reported outcomes. J Clin Oncol. 2018;36(11):1103-11.

54. Henry ML, Niu J, Zhang N, Giordano SH, Chavez-MacGregor M. Cardiotoxicity and cardiac monitoring among chemotherapy-treated breast cancer patients. JACC Cardiovasc Imaging. 2018;11(8):1084-93.

55. Chavez-MacGregor M, Niu J, Zhang N, Elting LS, Smith BD, Banchs J, et al. Cardiac monitoring during adjuvant trastuzumabbased chemotherapy among older patients with breast cancer. $\mathrm{J}$ Clin Oncol. 2015;33(19):2176.

56. Thuny F, Textoris J, Amara AB, El Filali A, Capo C, Habib G, et al. The gene expression analysis of blood reveals S100A11 and AQP9 as potential biomarkers of infective endocarditis. PLoS One. 2012;7(2):e31490
57. Alexandre J, Cautela J, Ederhy S, Damaj GL, Salem JE, Barlesi F, et al. Cardiovascular toxicity related to cancer treatment: a pragmatic approach to the American and European cardio-oncology guidelines. J Am Heart Assoc. 2020;9(18):e018403.

58. Leong DP, Cosman T, Alhussein MM, Kumar Tyagi N, Karampatos S, Barron CC, et al. Safety of continuing trastuzumab despite mild cardiotoxicity: a phase I trial. Cardio Oncology. 2019;1(1):1-10.

59. Rushton M, Lima I, Tuna M, Johnson C, Ivars J, Pritchard K, et al. Impact of stopping trastuzumab in early breast cancer: a population-based study in Ontario, Canada. JNCI J Natl Cancer Inst. 2020;112(12):1222-30.

60. Copeland-Halperin RS, Al-Sadawi M, Patil S, Liu JE, Steingart RM, Dang CT, et al. Early trastuzumab interruption and recurrence-free survival in ERBB2-positive breast cancer. JAMA Oncol. 2020;6(12):1971-2.

61. Kikuchi R, Shah NP, Dent SF. Strategies to prevent cardiovascular toxicity in breast cancer: is it ready for primetime? J Clin Med. 2020;9(4):896.

62. Vaduganathan M, Hirji SA, Qamar A, Bajaj N, Gupta A, Zaha VG, et al. Efficacy of neurohormonal therapies in preventing cardiotoxicity in patients with cancer undergoing chemotherapy. Cardio Oncology. 2019;1(1):54-65.

63. Gulati G, Heck SL, Ree AH, Hoffmann P, Schulz-Menger J, Fagerland MW, et al. Prevention of cardiac dysfunction during adjuvant breast cancer therapy (PRADA): a $2 \times 2$ factorial, randomized, placebo-controlled, double-blind clinical trial of candesartan and metoprolol. Eur Heart J. 2016;37(21):1671-80.

64. Guglin M, Krischer J, Tamura R, Fink A, Bello-Matricaria L, McCaskill-Stevens W, et al. Randomized trial of lisinopril versus carvedilol to prevent trastuzumab cardiotoxicity in patients with breast cancer. J Am Coll Cardiol. 2019;73(22):2859-68.

65. Boekhout AH, Gietema JA, Kerklaan BM, van Werkhoven ED, Altena R, Honkoop A, et al. Angiotensin II-receptor inhibition with candesartan to prevent trastuzumab-related cardiotoxic effects in patients with early breast cancer: a randomized clinical trial. JAMA Oncol. 2016;2(8):1030-7.

66. Pituskin E, Mackey JR, Koshman S, Jassal D, Pitz M, Haykowsky $\mathrm{MJ}$, et al. Multidisciplinary approach to novel therapies in cardiooncology research (MANTICORE 101-Breast): a randomized trial for the prevention of trastuzumab-associated cardiotoxicity. J Clin Oncol. 2017;35(8):870-7.

Publisher's Note Springer Nature remains neutral with regard to jurisdictional claims in published maps and institutional affiliations. 